\title{
Measurement of placental alkaline phosphatase activity in benign and malignant pleural effusions
}

\author{
R J Fergusson, J Fisken, M A McIntyre, J E Roulston, R C F Leonard
}

\begin{abstract}
The usefulness of placental alkaline phosphatase (PLAP) as a diagnostic marker of malignancy was assessed in pleural fluid from 60 patients with effusions. Pleural fluid PLAP activities were measured by an enzyme linked immunoassay (ELISA) using the two monoclonal antibodies H17E2 and H317. Similar values were found in groups of patients with primary bronchial tumours $(n=12)$, secondary malignancies $(n=23)$, and "benign" conditions $(n=25)$. The highest values were found in a small subgroup of patients with metastatic ovarian carcinoma. However, the production of this enzyme by normal lung makes the measurement of PLAP in pleural fluid unhelpful as a diagnostic aid to distinguish "benign" from malignant effusions.
\end{abstract}

(F Clin Pathol 1992;45:1114-1115)

Pleural effusions are common in clinical practice and may be produced by a wide variety of pathological processes. Elucidating the precise aetiology of these effusions may be difficult, and protracted investigations may be required. It is often important to be able to discriminate "benign" from malignant effusions; but the use of conventional cytological techniques to detect malignant cells has a sensitivity of less than $60 \% .^{12}$ Attempts to improve this have included the measurement of $\mathrm{pH}$, various biochemical variables, tumour associated antigens $^{2}$ and antibodies ${ }^{1}$ in pleural fluid. As yet no single technique with clinically useful specificity or sensitivity has emerged.

Placental-like alkaline phosphatase (PLAP) is an isoenzyme of the human syncytiotrophoblast which has been described in various cancer tissues as an oncodevelopmental protein $^{3}$ and shows potential as a marker of ovarian cancer. ${ }^{4}$ The enzyme has been found in normal lung ${ }^{3}$ and was first described in a patient with a primary bronchial carcinoma.

\section{Methods}

Samples of pleural fluid from 60 patients were studied. The patients were divided into three groups: 12 with primary bronchial carcinoma (four small cell, three squamous, two adenocarcinoma, two undifferentiated, one large cell), 23 with secondary tumour (seven breast, seven ovary, three lymphoma, three adenocarcinoma, two sarcoma, one leukaemia), and
25 with "benign" conditions (ten cardiac failure, three pneumonia, two rheumatoid, two empyema, one tuberculosis, one thromboembolism and six unknown). Patients in the "benign" group were followed up for one year to exclude any occult malignancy. The smoking habits of 52 of the 60 patients were noted. Samples $(40-500 \mathrm{ml})$ of pleural fluid were taken to the laboratory without delay following aspiration. The fluid was centrifuged at 2000 rpm for 10 minutes and cytological preparations were made for diagnostic purposes. The supernatant fluid was stored in $20 \mathrm{ml}$ aliquots at $-40^{\circ} \mathrm{C}$ before PLAP activity was measured. Pleural fluid PLAP values were measured by an enzyme linked immunoassay ${ }^{4}$ (ELISA) using the two monoclonal antibodies $\mathrm{H} 17 \mathrm{E} 2^{5}$ and H317. ${ }^{\circ}$ H17E2 recognises both PLAP and that which is produced by the lungs of smokers. H317 does not detect the latter. Statistical comparisons between groups were made using the Mann-Whitney $U$ test. The correlation between the two assays was evaluated using Spearman's coefficient.

\section{Results}

The mean pleural PLAP activities for each group using the two different monoclonal antibodies are outlined in the table. There was no significant difference between activities seen in patients with "benign" or malignant effusions. There was a wide scatter of values in each group (figure). The small subgroup of patients with a pleural effusion from secondary ovarian cancer had higher activities of pleural PLAP, although this was not significant.

There was a close correlation between the two assays using the two monoclonal antibodies $(r=0.7, p<0.001)$. Patients with malignant effusions (primary or secondary) had higher PLAP values with the H17E2 assay. The mean values in the two assays in patients with benign conditions were identical. Mean (SD) PLAP activities (H17E2 assay) were higher in current smokers: $0.76(0.8) \mathrm{IU} / 1$ ( $\mathrm{n}=$ 16) compared with non-smokers or ex-smokers of more than five years: $0.45(0.7) \mathrm{IU} / 1$ $(n=36)$. Values in smokers using the H317 antibody were not significantly lower $(0.69$ $(0 \cdot 7) \mathrm{IU} / 1)$.

Mean (SD) pleural PLAP activities (IU/l) for two different monoclonal antibodies

\begin{tabular}{lll}
\hline & H17E2 & $H 317$ \\
\hline Primary lung $(\mathrm{n}=12)$ & $0.68(0.88)$ & $0.48(0.47)$ \\
Secondary tumour $(\mathrm{n}=23)$ & $1.09(2.03)$ & $0.88(2.02)$ \\
Benign (n=25) & $0.54(0.95)$ & $0.54(0.86)$ \\
\hline
\end{tabular}




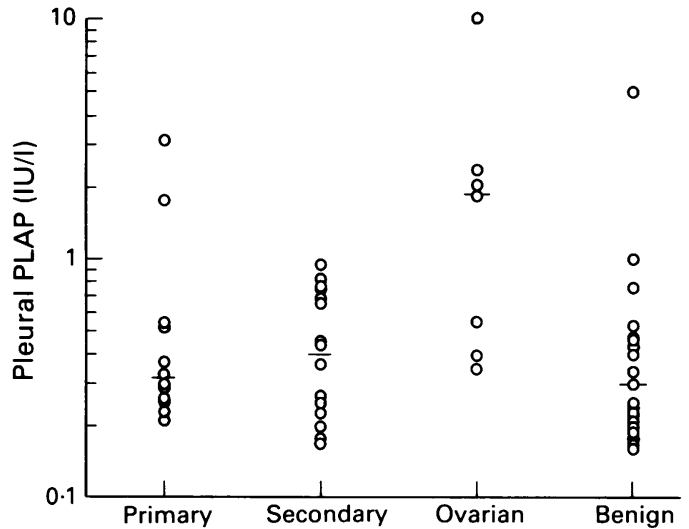

Scatter of pleural PLAP values in the groups studied (H17E2 antibody). Patients with ovarian carcinoma are shown as a separate group. Horizontal bars represent median values.

\section{Discussion}

Most studies evaluating PLAP as a tumour marker to date have involved estimation of enzyme activity in serum samples. This allows for easy monitoring of activities in outpatients. ${ }^{34}$ Of the few studies reporting PLAP measurements in other body fluids, there is evidence of raised values in the cerebrospinal fluid of patients with primary intracranial germinoma, ${ }^{7}$ in ascitic fluid of patients with ovarian carcinoma, ${ }^{8}$ and in peritoneal fluid from patients with endometriosis. ${ }^{9}$ In this last study samples obtained from healthy women also had detectable PLAP activity and in all studies there seemed to be a good correlation between the activities of PLAP in serum and in the body fluids sampled.

This is the first study assessing PLAP as a diagnostic marker of malignancy in pleural fluid. Our results have shown no difference between PLAP values in fluid from patients with "benign" and malignant effusions and no difference between primary and secondary tumours apart from higher values seen in a small subgroup of patients with ovarian cancer.

We found no difference in two assays using monoclonal antibodies with different specificities for PLAP and PLAP-like alkaline phos- phate. These results may be explained by previous reports of the relative abundance of PLAP in normal lung tissue compared with other normal tissues. ${ }^{3}$ The fact that the difference between PLAP values in normal and malignant extracts from one organ is narrowest in the lung and widest in the ovary ${ }^{3}$ may have facilitated the identification of a subgroup of patients with metastatic ovarian carcinoma.

Previous reports of measurement of PLAP in serum have shown a relation with smoking habits. ${ }^{10}$ We found a trend towards high activities of PLAP in pleural fluid from current smokers which is perhaps not surprising: there is evidence that this increased PLAP activity in smokers originates from the lung, ${ }^{10}$ indeed, some workers believe that PLAP may be a useful marker of acute lung injury. The results from this study suggest that PLAP is of no use as a diagnostic marker for malignancy when measured in pleural fluid.

1 Gosh AK, Mason DY, Spriggs AI. Immunocytochemical staining with monoclonal antibodies in cytologically "negative" serous effusions from patients with malignant disease. 7 Clin Pathol 1983;36:1150-3.

2 Mezger J, Permanetter W, Gerbes AL, Wilmanns W, Lamerz $R$. Tumour associated antigens in diagnosis of serous effusions. $\mathcal{F}$ Clin Pathol 1988;44:633-43.

3 Pollet DE, Nouwen EJ, Schelstraete JB, et al. Enzymeantigen immunoassay for human placental alkaline phosphatase in serum and tissue extracts and its application as a tumour marker. Clin Chem 1985;31:41-5.

4 Fisken J, Leonard RCF, Shaw G, Bowman A, Roulston JE. Serum placental-like alkaline phosphatase (PLAP): a novel combined enzyme-linked immunoassay for monitoring ovarian cancer. $\mathcal{F}$ Clin Pathol 1989;42:40- 5 .

5 Travers P, Bodmer WF. Preparation and characterisation of monoclonal antibodies against PLAP and other human trophoblast determinants. Int $\mathcal{F}$ Cancer 1984;33:633-41.

6 McLaughlin PJ, Cheng HM, Slade MB, Johnson PM. Expression on cultured human tumour cell lines of placental trophoblast membrane antigens and placental alkaline phosphatase defined by monoclonal antibodies. Int $\mathcal{F}$ Cancer 1982;30:21-6.

7 Shinoda J, Yamada H, Sakai N, et al. Placental alkaline phosphatase as a tumour marker for primary intracranial germinoma. $\Im$ Neurosurg 1988;68:710-20.

8 Koyama I, Stendahl U, Stigbrand T. Placental-like alkaline phosphatase from ovary and ascites of a patient with a malignant ovarian tumour. Clin Chim Acta 1989; malignant ovari

9 Kang JO, Hudak WA, Crowley WJ, Criswell BS. Placentaltype alkaline phosphatase in peritoneal fluid of women with endometriosis. Clin Chim Acta 1989;186:285-94

10 Kallioniemi OP, Nieminen MM, Lehtinen J, Veneskoski T, Kiovula T. Increased serum placental-like alkaline phosphatase activity in smokers originates from the lungs. Eur $\mathcal{F}$ Respir Dis 1987;71:170-6. 